\title{
Multiple positive solutions of boundary value problems for fractional order integro-differential equations in a Banach space
}

\author{
Ruijuan Liu ${ }^{1,2^{*}}$, Chunhai Kou ${ }^{3}$ and Ran Jin ${ }^{1}$
}

\section{"Correspondence:}

ruirui0516@163.com

${ }^{1}$ College of Information Science and

Technology, Donghua University,

Shanghai, 201620, China

${ }^{2}$ College of Fundamental Studies,

Shanghai University of Engineering

Science, Shanghai, 201620, China

Full list of author information is

available at the end of the article

\begin{abstract}
In this paper, we obtain the existence of multiple positive solutions of a boundary value problem for $\alpha$-order nonlinear integro-differential equations in a Banach space by means of fixed point index theory of completely continuous operators.

MSC: $26 \mathrm{~A} 33 ; 34 \mathrm{~B} 15$

Keywords: fractional order; integro-differential equation; measure of noncompactness; fixed point index; boundary value problem
\end{abstract}

\section{Introduction}

Fractional differential equations (FDEs) have been of great interest for the last three decades [1-11]. It is caused both by the intensive development of the theory of fractional calculus itself and by the applications of such constructions in the modeling of many phenomena in various fields of science and engineering. Indeed, we can find numerous applications in viscoelasticity [12], electrochemistry [13], control, porous media [14], etc. Therefore, the theory of FDEs has been developed very quickly. Many qualitative theories of FDEs have been obtained. Many important results can be found in [15-19] and references cited therein.

In this paper, we shall use the fixed point index theory of completely continuous operators to investigate the multiple positive solutions of a boundary value problem for a class of $\alpha$ order nonlinear integro-differential equations in a Banach space.

Let $E$ be a real Banach space, $P$ be a cone in $E$ and $P^{0}$ denote the interior points of $P$. A partial ordering in $E$ is introduced by $x \leq y$ if and only if $y-x \in P$. $P$ is said to be normal if there exists a positive constant $N$ such that $\theta \leq x \leq y$ implies $\|x\| \leq N\|y\|$, where $\theta$ denotes the zero element of $E$, and the smallest constant $N$ is called the normal constant of $P$.P is called solid if $P^{0}$ is nonempty. If $x \leq y$ and $x \neq y$, we write $x<y$. If $P$ is solid and $y-x \in P^{0}$, we write $x \ll y$. For details on cone theory, see [1].

For the application in the sequel, we first state the following lemmas and definitions which can be found in $[1,10,20]$.

Lemma 1.1 Let $P$ be a cone in a real Banach space $E$, and let $\Omega$ be a nonempty bounded open convex subset of $P$. Suppose that $A: \bar{\Omega} \rightarrow P$ is completely continuous and $A(\bar{\Omega}) \subset \Omega$, 
where $\bar{\Omega}$ denotes the closure of $\Omega$ in $P$. Then the fixed point index

$$
i(A, \Omega, P)=1 .
$$

Lemma 1.2 Let $P$ be a cone in a real Banach space $E$, and let $\Omega=\Omega_{1} \cup \Omega_{2}$, where $\Omega_{i}$ $(i=1,2)$ are nonempty bounded open convex subsets of $P$ and $\Omega_{1} \cap \Omega_{2}=\emptyset$. Suppose that $A: \bar{\Omega} \rightarrow P$ is a strict set contraction and $A(\bar{\Omega}) \subset \Omega$. Then

$$
i(A, \Omega, P)=i\left(A, \Omega_{1}, P\right)+i\left(A, \Omega_{2}, P\right) .
$$

Lemma 1.3 If $U \subset C[I, E]$ is bounded and equicontinuous, then $\alpha_{E}(U(t))$ is continuous on $I$, and set

$$
\alpha_{C}(U)=\max _{t \in I} \alpha_{E}(U(t)), \quad \alpha_{E}\left(\int_{I} u(t) \mathrm{d} t: u \in U\right) \leq \int_{I} \alpha_{E}(U(t)) \mathrm{d} t,
$$

where $I=[a, b], U(t)=\{u(t): u \in U\}$.

Definition 1.1 The fractional integral of order $\alpha>0$ of a function $f:(0, \infty) \rightarrow \mathbb{R}$ is given by

$$
I_{0+}^{\alpha} f(t)=\frac{1}{\Gamma(\alpha)} \int_{0}^{t}(t-s)^{\alpha-1} f(s) \mathrm{d} s
$$

provided the right-hand side is pointwise defined on $(0, \infty)$.

Definition 1.2 The fractional derivative of order $\alpha>0$ of a function $f:(0, \infty) \rightarrow \mathbb{R}$ is given by

$$
D_{0+}^{\alpha} f(t)=\frac{1}{\Gamma(n-\alpha)}\left(\frac{\mathrm{d}}{\mathrm{d} t}\right)^{n} \int_{0}^{t} \frac{f(s)}{(t-s)^{\alpha-n+1}} \mathrm{~d} s,
$$

where $n=[\alpha]+1$, provided the right-hand side is pointwise defined on $(0, \infty)$.

Lemma 1.4 Let $\alpha>0$, then

$$
I_{0+}^{\alpha} D_{0+}^{\alpha} x(t)=x(t)+c_{1} t^{\alpha-1}+c_{2} t^{\alpha-2}+\cdots+c_{n} t^{\alpha-n}
$$

for some $c_{i} \in E, i=0,1,2, \ldots, n-1, n=-[-\alpha]$.

In this article, let $J=[0,+\infty), B C[J, E]=\left\{u \in C[J, E]: \sup _{t \in J} \frac{\|u(t)\|}{1+t^{\alpha-1}}<\infty\right\}$. It is easy to see that $B C[, E]$ is a Banach space with the norm

$$
\|u\|_{B}=\sup _{t \in J} \frac{\|u(t)\|}{1+t^{\alpha-1}} .
$$

Consider the boundary value problem (BVP) for a fractional nonlinear integro-differential equation of mixed type in $E$ :

$$
\left\{\begin{array}{l}
D_{0}^{\alpha} u(t)+f(t, u(t),(T u)(t),(S u)(t))=\theta \quad \forall t \in J, \\
u(0)=u^{\prime}(0)=\theta, \quad D_{0}^{\alpha-1} u(+\infty)=\sum_{i=1}^{m} \beta_{i} u\left(\eta_{i}\right),
\end{array}\right.
$$


where $D_{0}^{\alpha}$ is the standard Riemann-Liouville fractional derivative of order $2<\alpha<3, f \in$ $C[J \times P \times P \times P, P], \beta_{i}>0(i=1,2, \ldots, m), 0<\eta_{1}<\eta_{2}<\cdots<\eta_{m}, \sum_{i=1}^{m} \beta_{i} \eta_{i}^{\alpha-1}<\Gamma(\alpha)$ and

$$
(T u)(t)=\int_{0}^{t} K(t, s) u(s) \mathrm{d} s, \quad(S u)(t)=\int_{0}^{+\infty} H(t, s) u(s) \mathrm{d} s,
$$

$K \in C\left[D, R^{+}\right], D=\{(t, s) \in J \times J: t \geq s\}, H \in C\left[J \times J, R^{+}\right], R^{+}$denotes the set of all nonnegative real numbers.

\section{Several lemmas}

To establish the existence of multiple positive solutions in $B C[J, P]$ of (1), let us list the following assumptions.

$\left(\mathrm{H}_{1}\right) \quad k^{*}=\sup _{t \in J} \int_{0}^{t} K(t, s) \mathrm{d} s<\infty, h^{*}=\sup _{t \in J} \frac{1}{1+t^{\alpha-1}} \int_{0}^{+\infty} H(t, s)\left(1+s^{\alpha-1}\right) \mathrm{d} s<\infty$, $\int_{0}^{+\infty}\left(H\left(t^{\prime}, s\right)-H(t, s)\right)\left(1+s^{\alpha-1}\right) \mathrm{d} s \rightarrow 0$, as $t^{\prime} \rightarrow t(t \in J)$.

$\left(\mathrm{H}_{2}\right)$ There exist $a, b \in C\left[, R^{+}\right]$and $g \in C\left[J \times J \times J, R^{+}\right]$such that

$$
\|f(t, u, v, w)\| \leq a(t)+b(t) g(\|u\|,\|v\|,\|w\|) \quad \forall t \in J, u, v, w \in P .
$$

$\left(\mathrm{H}_{3}\right)$ There exists $c \in C\left[U, R^{+}\right]$such that

$$
\frac{\|f(t, u, v, w)\|}{c(t)(\|u\|+\|v\|+\|w\|)} \rightarrow 0, \quad \text { as } u, v, w \in P,\|u\|+\|v\|+\|w\| \rightarrow \infty
$$

uniformly for $t \in J$, and

$$
c^{*}=\int_{0}^{+\infty} c(t)\left(1+t^{\alpha-1}\right) \mathrm{d} t<\infty .
$$

$\left(\mathrm{H}_{4}\right)$ There exists $d \in C\left[J, R^{+}\right]$such that

$$
\frac{\left\|f\left(t,\left(1+t^{\alpha-1}\right) u,\left(1+t^{\alpha-1}\right) v,\left(1+t^{\alpha-1}\right) w\right)\right\|}{d(t)(\|u\|+\|v\|+\|w\|)} \rightarrow 0, \quad \text { as } u, v, w \in P,\|u\|+\|v\|+\|w\| \rightarrow 0
$$

uniformly for $t \in J$, and

$$
d^{*}=\int_{0}^{+\infty} d(t) \mathrm{d} t<\infty
$$

$\left(\mathrm{H}_{5}\right)$ For any $t \in J$ and $r>0, f\left(t, P_{r}, P_{r}, P_{r}\right)=\left\{f(t, u, v, w): u, v, w \in P_{r}\right\}$ is relatively compact in $E$, where $P_{r}=\{u \in P:\|u\| \leq r\}$.

$\left(\mathrm{H}_{6}\right) P$ is normal and solid, and there exist $u_{0} \gg \theta, 0<t_{*}<t^{*}<\infty$ and $\sigma \in C\left[I, R^{+}\right]$such that

$$
f(t, u, v, w) \geq \sigma(t) u_{0} \quad \forall t \in I, u \geq u_{0}, v \geq \theta, w \geq \theta
$$

and

$$
\int_{t_{*}}^{t^{*}} \gamma(s) \sigma(s)>1
$$

where $I=\left[t_{*}, t^{*}\right], \gamma(s)=\min _{t \in I} G(t, s)$. 
$\left(\mathrm{H}_{7}\right)$ There exist $u_{0}>\theta, 0<t_{*}<t^{*}<\infty$ and $\sigma \in C\left[I, R^{+}\right]$such that

$$
f(t, u, v, w) \geq \sigma(t) u_{0} \quad \forall t \in I, u \geq u_{0}, v \geq \theta, w \geq \theta
$$

and

$$
\int_{t_{*}}^{t^{*}} \gamma(s) \sigma(s) \geq 1
$$

where $I=\left[t_{*}, t^{*}\right], \gamma(s)=\min _{t \in I} G(t, s)$.

Remark 2.1 It is clear that $\left(\mathrm{H}_{5}\right)$ is satisfied automatically when $E$ is finite dimensional.

Remark 2.2 It is clear that assumption $\left(\mathrm{H}_{7}\right)$ is weaker than assumption $\left(\mathrm{H}_{6}\right)$.

We shall reduce BVP (1) to an integral equation in $E$. To this end, we first consider the operator $A$ defined by

$$
\begin{aligned}
(A u)(t)= & -\frac{1}{\Gamma(\alpha)} \int_{0}^{t}(t-s)^{\alpha-1} f(s, u(s),(T u)(s),(S u)(s)) \mathrm{d} s \\
& -\frac{\lambda}{\Gamma(\alpha)} \sum_{i=1}^{m} \beta_{i} t^{\alpha-1} \int_{0}^{\eta_{i}}\left(\eta_{i}-s\right)^{\alpha-1} f(s, u(s),(T u)(s),(S u)(s)) \mathrm{d} s \\
& +\lambda t^{\alpha-1} \int_{0}^{+\infty} f(s, u(s),(T u)(s),(S u)(s)) \mathrm{d} s,
\end{aligned}
$$

where $\lambda=\frac{1}{\Gamma(\alpha)-\sum_{i=1}^{m} \beta_{i} \eta_{i}^{\alpha-1}}$.

In our main results, we make use of the following lemmas.

Lemma 2.1 Let assumption $\left(\mathrm{H}_{1}\right)$ be satisfied, then the operators $T$ and $S$ defined by (2) are bounded linear operators from $B C[J, E]$ into $B C[, P]$, and

$$
\|T\| \leq k^{*}, \quad\|S\| \leq h^{*} .
$$

Moreover,

$$
T: B C[J, P] \rightarrow B C[J, P], \quad S: B C[J, P] \rightarrow B C[J, P] .
$$

Proof Inequalities (4) follow from two simple inequalities:

$$
\begin{aligned}
\frac{\|(T u)(t)\|}{1+t^{\alpha-1}} & \leq \int_{0}^{t} K(t, s) \frac{1+s^{\alpha-1}}{1+t^{\alpha-1}} \frac{\|u(s)\|}{1+s^{\alpha-1}} \mathrm{~d} s \leq k^{*}\|u\|_{B}, \\
\frac{\|(S u)(t)\|}{1+t^{\alpha-1}} & \leq \int_{0}^{+\infty} H(t, s) \frac{1+s^{\alpha-1}}{1+t^{\alpha-1}} \frac{\|u(s)\|}{1+s^{\alpha-1}} \mathrm{~d} s \leq h^{*}\|u\|_{B},
\end{aligned}
$$

and (5) is obvious.

Lemma 2.2 Let assumptions $\left(\mathrm{H}_{1}\right),\left(\mathrm{H}_{2}\right)$ and $\left(\mathrm{H}_{3}\right)$ be satisfied, then the operator A defined by (3) is a continuous operator from $B C[, E]$ into $B C[J, E]$. 
Proof Let

$$
\varepsilon_{1}=\frac{1}{2\left(1+k^{*}+h^{*}\right)}\left[\left(\frac{1}{\Gamma(\alpha)}+\lambda\right) c^{*}+\frac{\lambda}{\Gamma(\alpha)} \sum_{i=1}^{m} \beta_{i} \int_{0}^{\eta_{i}}\left(\eta_{i}-s\right)^{\alpha-1}(a(s)+M b(s)) \mathrm{d} s\right]^{-1},
$$

where $\lambda$ is defined in the operator $A$.

By virtue of assumptions $\left(\mathrm{H}_{2}\right)$ and $\left(\mathrm{H}_{3}\right)$, there exists an $R>0$ such that

$$
\begin{gathered}
\|f(t, u, v, w)\| \leq \varepsilon_{1} c(t)(\|u\|+\|v\|+\|w\|) \\
\forall t \in J, u, v, w \in P,\|u\|+\|v\|+\|w\|>R
\end{gathered}
$$

and

$$
\begin{aligned}
& \|f(t, u, v, w)\| \leq a(t)+M b(t) \\
& \forall t \in J, u, v, w \in P,\|u\|+\|v\|+\|w\| \leq R,
\end{aligned}
$$

where

$$
M=\max \left\{g\left(x_{1}, x_{2}, x_{3}\right): 0 \leq x_{1}, x_{2}, x_{3} \leq R\right\} .
$$

It follows from (6) and (7) that for $t \in J, u, v, w \in P$, we have

$$
\|f(t, u, v, w)\| \leq \varepsilon_{1} c(t)(\|u\|+\|v\|+\|w\|)+a(t)+M b(t) .
$$

Let $u \in B C[$,P], we have, by (8) and Lemma 2.1,

$$
\|f(t, u,(T u)(t),(S u)(t))\| \leq \varepsilon_{1} c(t)\left(1+t^{\alpha-1}\right)\left(1+k^{*}+h^{*}\right)\|u\|_{B}+a(t)+M b(t),
$$

which implies the convergence of the infinite integral

$$
\int_{0}^{+\infty} f(t, u,(T u)(t),(S u)(t)) \mathrm{d} s
$$

and

$$
\int_{0}^{+\infty}\|f(t, u,(T u)(t),(S u)(t))\| \mathrm{d} s \leq c^{*} \varepsilon_{1}\left(1+k^{*}+h^{*}\right)\|u\|_{B}+a^{*}+M b^{*} .
$$

Thus, we have, by (3), (9) and (10),

$$
\begin{aligned}
\frac{\|(A u)(t)\|}{1+t^{\alpha-1}} \leq & \frac{1}{\Gamma(\alpha)} \int_{0}^{t} \frac{(t-s)^{\alpha-1}}{1+t^{\alpha-1}}\|f(s, u(s),(T u)(s),(S u)(s))\| \mathrm{d} s \\
& +\frac{\lambda}{\Gamma(\alpha)} \sum_{i=1}^{m} \beta_{i} \frac{t^{\alpha-1}}{1+t^{\alpha-1}} \int_{0}^{\eta_{i}}\left(\eta_{i}-s\right)^{\alpha-1}\|f(s, u(s),(T u)(s),(S u)(s))\| \mathrm{d} s \\
& +\frac{\lambda t^{\alpha-1}}{1+t^{\alpha-1}} \int_{0}^{+\infty}\|f(s, u(s),(T u)(s),(S u)(s))\| \mathrm{d} s
\end{aligned}
$$




$$
\begin{aligned}
\leq & \left(\frac{1}{\Gamma(\alpha)}+\lambda\right) \int_{0}^{+\infty}\|f(s, u(s),(T u)(s),(S u)(s))\| \mathrm{d} s \\
& +\frac{\lambda}{\Gamma(\alpha)} \sum_{i=1}^{m} \beta_{i} \int_{0}^{\eta_{i}}\left(\eta_{i}-s\right)^{\alpha-1}\|f(s, u(s),(T u)(s),(S u)(s))\| \mathrm{d} s \\
\leq & \left(\frac{1}{\Gamma(\alpha)}+\lambda\right)\left(c^{*} \varepsilon_{1}\left(1+k^{*}+h^{*}\right)\|u\|_{B}+a^{*}+M b^{*}\right) \\
& +\frac{\lambda \varepsilon_{1}}{\Gamma(\alpha)} \sum_{i=1}^{m} \beta_{i} \int_{0}^{\eta_{i}}\left(\eta_{i}-s\right)^{\alpha-1} \varepsilon_{1} c(s)\left(1+s^{\alpha-1}\right)\left(1+k^{*}+h^{*}\right)\|u\|_{B} \mathrm{~d} s \\
& +\frac{\lambda}{\Gamma(\alpha)} \sum_{i=1}^{m} \beta_{i} \int_{0}^{\eta_{i}}\left(\eta_{i}-s\right)^{\alpha-1}(a(s)+M b(s)) \mathrm{d} s \\
\leq & \frac{1}{2}\|u\|_{B}+\left(\frac{1}{\Gamma(\alpha)}+\lambda\right)\left(a^{*}+M b^{*}\right) \\
& +\frac{\lambda}{\Gamma(\alpha)} \sum_{i=1}^{m} \beta_{i} \int_{0}^{\eta_{i}}\left(\eta_{i}-s\right)^{\alpha-1}(a(s)+M b(s)) \mathrm{d} s .
\end{aligned}
$$

It follows from (11) that

$$
\begin{aligned}
\|A u\|_{B} \leq & \frac{1}{2}\|u\|_{B}+\left(\frac{1}{\Gamma(\alpha)}+\lambda\right)\left(a^{*}+M b^{*}\right) \\
& +\frac{\lambda}{\Gamma(\alpha)} \sum_{i=1}^{m} \beta_{i} \int_{0}^{\eta_{i}}\left(\eta_{i}-s\right)^{\alpha-1}(a(s)+M b(s)) \mathrm{d} s
\end{aligned}
$$

Thus, we have $A(B C[J, E]) \subset B C[J, E]$.

Finally, we show that $A$ is continuous. Let $u_{n}, \tilde{u} \in B C[J, E],\left\|u_{n}-\tilde{u}\right\|_{B} \rightarrow 0(n \rightarrow \infty)$. Then $r=\sup _{n}\left\|u_{n}\right\|<\infty$ and $\|\tilde{u}\|_{B} \leq r$. By (3), we have

$$
\begin{aligned}
&\left\|\frac{\left(A u_{n}\right)(t)}{1+t^{\alpha-1}}-\frac{(A \tilde{u})(t)}{1+t^{\alpha-1}}\right\| \\
& \leq \int_{0}^{t} \frac{(t-s)^{\alpha-1}}{1+t^{\alpha-1}}\left\|f\left(s, u_{n}(s),\left(T u_{n}\right)(s),\left(S u_{n}\right)(s)\right)-f(s, \tilde{u}(s),(T \tilde{u})(s),(S \tilde{u})(s))\right\| \mathrm{d} s \\
& \quad+\frac{\lambda}{\Gamma(\alpha)} \sum_{i=1}^{m} \beta_{i} \frac{t^{\alpha-1}}{1+t^{\alpha-1}} \int_{0}^{\eta_{i}}\left(\eta_{i}-s\right)^{\alpha-1} \| f\left(s, u_{n}(s),\left(T u_{n}\right)(s),\left(S u_{n}\right)(s)\right) \\
& \quad-f(s, \tilde{u}(s),(T \tilde{u})(s),(S \tilde{u})(s)) \| \mathrm{d} s \\
& \quad+\frac{\lambda t^{\alpha-1}}{1+t^{\alpha-1}} \int_{0}^{+\infty}\left\|f\left(s, u_{n}(s),\left(T u_{n}\right)(s),\left(S u_{n}\right)(s)\right)-f(s, \tilde{u}(s),(T \tilde{u})(s),(S \tilde{u})(s))\right\| \mathrm{d} s \\
& \leq\left.\frac{1}{\Gamma(\alpha)}+\lambda\right) \int_{0}^{+\infty}\left\|f\left(s, u_{n}(s),\left(T u_{n}\right)(s),\left(S u_{n}\right)(s)\right)-f(s, \tilde{u}(s),(T \tilde{u})(s),(S \tilde{u})(s))\right\| \mathrm{d} s \\
&+\frac{\lambda}{\Gamma(\alpha)} \sum_{i=1}^{m} \beta_{i} \int_{0}^{\eta_{i}}\left(\eta_{i}-s\right)^{\alpha-1} \| f\left(s, u_{n}(s),\left(T u_{n}\right)(s),\left(S u_{n}\right)(s)\right) \\
&-f(s, \tilde{u}(s),(T \tilde{u})(s),(S \tilde{u})(s)) \| \mathrm{d} s .
\end{aligned}
$$


It is clear that

$$
f\left(t, u_{n}(t),\left(T u_{n}\right)(t),\left(S u_{n}\right)(t)\right) \rightarrow f(t, \tilde{u}(t),(T \tilde{u})(t),(S \tilde{u})(t)), \quad n \rightarrow \infty,
$$

and by (9),

$$
\begin{aligned}
& \left\|f\left(t, u_{n}(t),\left(T u_{n}\right)(t),\left(S u_{n}\right)(t)\right)-f(t, \tilde{u}(t),(T \tilde{u})(t),(S \tilde{u})(t))\right\| \\
& \leq 2 \varepsilon_{1} c(t)\left(1+t^{\alpha-1}\right)\left(1+k^{*}+h^{*}\right)\|u\|_{B}+2 a(t)+2 M b(t)=\mu(t) \\
& \quad \forall t \in J, n=1,2,3, \ldots, \mu \in L\left[J, R^{+}\right] .
\end{aligned}
$$

It follows from (15) and (16) and the dominated convergence theorem that

$$
\lim _{n \rightarrow \infty} \int_{0}^{+\infty}\left\|f\left(t, u_{n}(t),\left(T u_{n}\right)(t),\left(S u_{n}\right)(t)\right)-f(t, \tilde{u}(t),(T \tilde{u})(t),(S \tilde{u})(t))\right\| \mathrm{d} s=0
$$

and

$$
\begin{aligned}
& \lim _{n \rightarrow \infty} \int_{0}^{\eta_{i}}\left(\eta_{i}-s\right)^{\alpha-1}\left\|f\left(t, u_{n}(t),\left(T u_{n}\right)(t),\left(S u_{n}\right)(t)\right)-f(t, \tilde{u}(t),(T \tilde{u})(t),(S \tilde{u})(t))\right\| \mathrm{d} s \\
& \quad=0, \quad i=1,2, \ldots, m .
\end{aligned}
$$

It follows from (14), (17) and (18) that $\left\|A u_{n}-A \tilde{u}\right\|_{B} \rightarrow 0(n \rightarrow \infty)$, and the continuity of $A$ is proved.

Lemma 2.3 Let assumptions $\left(\mathrm{H}_{1}\right),\left(\mathrm{H}_{2}\right)$ and $\left(\mathrm{H}_{3}\right)$ be satisfied, then $u \in B C[J, E]$ is a solution of $B V P(1)$ if and only if $u \in B C[J, E]$ is a solution of the following integral equation:

$$
\begin{aligned}
u(t)= & -\frac{1}{\Gamma(\alpha)} \int_{0}^{t}(t-s)^{\alpha-1} f(s, u(s),(T u)(s),(S u)(s)) \mathrm{d} s \\
& -\frac{\lambda}{\Gamma(\alpha)} \sum_{i=1}^{m} \beta_{i} t^{\alpha-1} \int_{0}^{\eta_{i}}\left(\eta_{i}-s\right)^{\alpha-1} f(s, u(s),(T u)(s),(S u)(s)) \mathrm{d} s \\
& +\lambda t^{\alpha-1} \int_{0}^{+\infty} f(s, u(s),(T u)(s),(S u)(s)) \mathrm{d} s,
\end{aligned}
$$

i.e., $u$ is a fixed point of the operator $A$ defined by (3) in $B C[J, E]$.

Proof If $u \in B C[J, E]$ is a solution of BVP (1), then by applying Lemma 1.4 we reduce $D_{0}^{\alpha} u(t)+f(t, u(t),(T u)(t),(S u)(t))=\theta$ to an equivalent integral equation

$$
u(t)=-I_{0+}^{\alpha} f(t, u(t),(T u)(t),(S u)(t))+c_{1} t^{\alpha-1}+c_{2} t^{\alpha-2}+c_{3} t^{\alpha-3}
$$

for some $c_{1}, c_{2}, c_{3}$. (20) can be rewritten

$$
\begin{aligned}
u(t)= & -\frac{1}{\Gamma(\alpha)} \int_{0}^{t}(t-s)^{\alpha-1} f(s, u(s),(T u)(s),(S u)(s)) \mathrm{d} s \\
& +c_{1} t^{\alpha-1}+c_{2} t^{\alpha-2}+c_{3} t^{\alpha-3} .
\end{aligned}
$$


By $u(0)=u^{\prime}(0)=\theta$, we have

$$
c_{2}=c_{3}=0 \text {. }
$$

By $D_{0}^{\alpha-1} u(+\infty)=\sum_{i=1}^{m} \beta_{i} u\left(\eta_{i}\right)$, we obtain

$$
\begin{aligned}
c_{1}= & \lambda \int_{0}^{+\infty} f(s, u(s),(T u)(s),(S u)(s)) \mathrm{d} s \\
& -\frac{\lambda}{\Gamma(\alpha)} \sum_{i=1}^{m} \beta_{i} \int_{0}^{\eta_{i}}\left(\eta_{i}-s\right)^{\alpha-1} f(s, u(s),(T u)(s),(S u)(s)) \mathrm{d} s .
\end{aligned}
$$

Now, substituting (22) and (23) into (21), we see that $u(t)$ satisfies integral equation (19). Conversely, if $u$ is a solution of (19), the direct differentiation of (19) gives

$$
\begin{aligned}
u^{\prime}(t)= & -\frac{1}{\Gamma(\alpha-1)} \int_{0}^{t}(t-s)^{\alpha-2} f(s, u(s),(T u)(s),(S u)(s)) \mathrm{d} s \\
& -\frac{\lambda}{\Gamma(\alpha-1)} \sum_{i=1}^{m} \beta_{i} t^{\alpha-2} \int_{0}^{\eta_{i}}\left(\eta_{i}-s\right)^{\alpha-1} f(s, u(s),(T u)(s),(S u)(s)) \mathrm{d} s \\
& +\lambda(\alpha-1) t^{\alpha-2} \int_{0}^{+\infty} f(s, u(s),(T u)(s),(S u)(s)) \mathrm{d} s
\end{aligned}
$$

and

$$
D_{0+}^{\alpha-1} u(t)=\int_{0}^{t} f(s, u(s),(T u)(s),(S u)(s)) \mathrm{d} s .
$$

Consequently, $u \in B C[J, E]$, and by (19), (24) and (25), it is easy to see that $u(t)$ satisfies BVP (1).

Lemma 2.4 Integral equation (19) can be expressed as

$$
u(t)=\int_{0}^{+\infty} G(t, s) f(s, u(s),(T u)(s),(S u)(s)) \mathrm{d} s
$$

and $G(t, s)>0$ for any $t, s \in(0, \infty)$, where

$$
G(t, s)=\left\{\begin{array}{l}
\frac{-(t-s)^{\alpha-1}\left(\Gamma(\alpha)-\sum_{i=1}^{m} \beta_{i} \eta_{i}^{\alpha-1}\right)-\sum_{i=j}^{m} \beta_{i} t^{\alpha-1}\left(\eta_{i}-s\right)^{\alpha-1}+\Gamma(\alpha) t^{\alpha-1}}{\left(\Gamma(\alpha)-\sum_{i=1}^{m} \beta_{i} \eta_{i}^{\alpha-1}\right) \Gamma(\alpha)}, \\
\eta_{k-1} \leq t \leq \eta_{k}, \eta_{j-1} \leq s \leq \eta_{j}, k=1,2, \ldots, m, \\
j=1,2, \ldots, k-1 \text { or } \\
\eta_{k-1} \leq t \leq \eta_{k}, s \leq t, k=1,2, \ldots, m ; \\
\frac{-\sum_{i=j}^{m} \beta_{i} t^{\alpha-1}\left(\eta_{i}-s\right)^{\alpha-1}+\Gamma(\alpha) t^{\alpha-1}}{\left(\Gamma(\alpha)-\sum_{i=1}^{m} \beta_{i} \eta_{i}^{\alpha-1}\right) \Gamma(\alpha)}, \\
\eta_{k-1} \leq t \leq \eta_{k}, \eta_{j-1} \leq s \leq \eta_{j}, k=1,2, \ldots, m, \\
j=k+1, \ldots, m \text { or } \\
\eta_{k-1} \leq t \leq \eta_{k}, t \leq s, k=1,2, \ldots, m ; \\
\frac{-(t-s)^{\alpha-1}\left(\Gamma(\alpha)-\sum_{i=1}^{m} \beta_{i} \eta_{i}^{\alpha-1}\right)+\Gamma(\alpha) t^{\alpha-1}}{\left(\Gamma(\alpha)-\sum_{i=1}^{m} \beta_{i} \eta_{i}^{\alpha-1}\right) \Gamma(\alpha)}, \quad \eta_{m} \leq s \leq t ; \\
\frac{t^{\alpha-1}}{\Gamma(\alpha)-\sum_{i=1}^{m} \beta_{i} \eta_{i}^{\alpha-1}}, \quad t \leq \eta_{m} \leq s \text { or } \eta_{m} \leq t \leq s .
\end{array}\right.
$$


Proof Let $h(t)=f(t, u(t),(T u)(t),(S u)(t))$. For $t \leq \eta_{1}$, one has

$$
\begin{aligned}
u(t)= & -\frac{1}{\Gamma(\alpha)} \int_{0}^{t}(t-s)^{\alpha-1} h(s) \mathrm{d} s \\
& -\frac{\lambda}{\Gamma(\alpha)} \beta_{1} t^{\alpha-1}\left(\int_{0}^{t}\left(\eta_{1}-s\right)^{\alpha-1} h(s) \mathrm{d} s+\int_{t}^{\eta_{1}}\left(\eta_{1}-s\right)^{\alpha-1} h(s) \mathrm{d} s\right) \\
& -\frac{\lambda}{\Gamma(\alpha)} \beta_{2} t^{\alpha-1}\left(\int_{0}^{t}\left(\eta_{2}-s\right)^{\alpha-1} h(s) \mathrm{d} s+\int_{t}^{\eta_{1}}\left(\eta_{2}-s\right)^{\alpha-1} h(s) \mathrm{d} s\right. \\
& \left.+\int_{\eta_{1}}^{\eta_{2}}\left(\eta_{2}-s\right)^{\alpha-1} h(s) \mathrm{d} s\right) \\
& \cdots \\
& -\frac{\lambda}{\Gamma(\alpha)} \beta_{m} t^{\alpha-1}\left(\int_{0}^{t}\left(\eta_{m}-s\right)^{\alpha-1} h(s) \mathrm{d} s+\int_{t}^{\eta_{1}}\left(\eta_{m}-s\right)^{\alpha-1} h(s) \mathrm{d} s\right. \\
& \left.+\cdots+\int_{\eta_{m-1}}^{\eta_{m}}\left(\eta_{m}-s\right)^{\alpha-1} h(s) \mathrm{d} s\right) \\
& +\lambda t^{\alpha-1}\left(\int_{0}^{t} h(s) \mathrm{d} s+\int_{t}^{\eta_{1}} h(s) \mathrm{d} s+\int_{\eta_{1}}^{\eta_{2}} h(s) \mathrm{d} s\right. \\
& \left.+\cdots+\int_{\eta_{m-1}}^{\eta_{m}} h(s) \mathrm{d} s+\int_{\eta_{m}}^{+\infty} h(s) \mathrm{d} s\right) \\
& +\infty(t, s) h(s) \mathrm{d} s,
\end{aligned}
$$

$0<s \leq t$

$$
\begin{aligned}
G(t, s) & =\frac{\lambda}{\Gamma(\alpha)}\left[-(t-s)^{\alpha-1}\left(\Gamma(\alpha)-\sum_{i=1}^{m} \beta_{i} \eta_{i}^{\alpha-1}\right)-\sum_{i=1}^{m} \beta_{i}\left(\eta_{i}-s\right)^{\alpha-1} t^{\alpha-1}+\Gamma(\alpha) t^{\alpha-1}\right] \\
& \geq \frac{\lambda}{\Gamma(\alpha)}\left[-t^{\alpha-1}\left(\Gamma(\alpha)-\sum_{i=1}^{m} \beta_{i} \eta_{i}^{\alpha-1}\right)-\sum_{i=1}^{m} \beta_{i}\left(\eta_{i}-s\right)^{\alpha-1} t^{\alpha-1}+\Gamma(\alpha) t^{\alpha-1}\right] \\
& =\frac{\lambda}{\Gamma(\alpha)} \sum_{i=1}^{m} \beta_{i}\left(\eta_{i}^{\alpha-1}-\left(\eta_{i}-s\right)^{\alpha-1}\right) t^{\alpha-1}>0,
\end{aligned}
$$

$0<t \leq s \leq \eta_{1}$

$$
\begin{aligned}
G(t, s) & =\frac{\lambda}{\Gamma(\alpha)}\left[-\sum_{i=1}^{m} \beta_{i}\left(\eta_{i}-s\right)^{\alpha-1} t^{\alpha-1}+\Gamma(\alpha) t^{\alpha-1}\right] \\
& \geq \frac{\lambda}{\Gamma(\alpha)}\left(\Gamma(\alpha)-\sum_{i=1}^{m} \beta_{i} \eta_{i}^{\alpha-1}\right) t^{\alpha-1} \geq 0,
\end{aligned}
$$

$\eta_{j-1} \leq s \leq \eta_{j}, j=2,3, \ldots, m$

$$
\begin{aligned}
G(t, s) & =\frac{\lambda}{\Gamma(\alpha)}\left[-\sum_{i=j}^{m} \beta_{i}\left(\eta_{i}-s\right)^{\alpha-1} t^{\alpha-1}+\Gamma(\alpha) t^{\alpha-1}\right] \\
& \geq \frac{\lambda}{\Gamma(\alpha)}\left(\Gamma(\alpha)-\sum_{i=j}^{m} \beta_{i} \eta_{i}^{\alpha-1}\right) t^{\alpha-1}>0,
\end{aligned}
$$


$\eta_{m} \leq s$

$$
G(t, s)=\frac{\lambda}{\Gamma(\alpha)} t^{\alpha-1}>0
$$

By simple calculation, we can prove the rest of the lemma.

Lemma 2.5 Let assumptions $\left(\mathrm{H}_{1}\right),\left(\mathrm{H}_{2}\right)$ and $\left(\mathrm{H}_{3}\right)$ be satisfied, and let $U$ be a bounded subset of $B C[J, E]$. Then $\left\{\frac{(A u)(t)}{1+t_{2}^{\alpha-1}}: u \in U\right\}$ is equicontinuous on any finite subinterval of $J$, and for any given $\varepsilon>0$, there exists $\tau>0$ such that

$$
\left\|\frac{A u\left(t_{1}\right)}{1+t_{1}^{\alpha-1}}-\frac{A u\left(t_{2}\right)}{1+t_{2}^{\alpha-1}}\right\|<\varepsilon
$$

uniformly with respect to $u \in U$, as $t_{1}, t_{2} \geq \tau$.

Proof For $u \in U, t_{1}<t_{2}$, by using (3), we have

$$
\begin{aligned}
& \left\|\frac{A u\left(t_{1}\right)}{1+t_{1}^{\alpha-1}}-\frac{A u\left(t_{2}\right)}{1+t_{2}^{\alpha-1}}\right\| \\
& \leq \frac{1}{\Gamma(\alpha)} \int_{0}^{t_{1}}\left|\frac{\left(t_{1}-s\right)^{\alpha-1}}{1+t_{1}^{\alpha-1}}-\frac{\left(t_{2}-s\right)^{\alpha-1}}{1+t_{2}^{\alpha-1}}\right|\|f(s, u(s),(T u)(s),(S u)(s))\| \mathrm{d} s \\
& \quad+\frac{1}{\Gamma(\alpha)} \int_{t_{1}}^{t_{2}} \frac{\left(t_{2}-s\right)^{\alpha-1}}{1+t_{2}^{\alpha-1}}\|f(s, u(s),(T u)(s),(S u)(s))\| \mathrm{d} s \\
& \quad+\left|\frac{t_{1}^{\alpha-1}}{1+t_{1}^{\alpha-1}}-\frac{t_{2}^{\alpha-1}}{1+t_{2}^{\alpha-1}}\right|\left(\lambda \int_{0}^{+\infty}\|f(t, u,(T u)(t),(S u)(t))\| \mathrm{d} s\right. \\
& \left.\quad+\frac{1}{\Gamma(\alpha)} \sum_{i=1}^{m} \beta_{i} \int_{0}^{\eta_{i}}\left(\eta_{i}-s\right)^{\alpha-1}\|f(s, u(s),(T u)(s),(S u)(s))\| \mathrm{d} s\right) .
\end{aligned}
$$

This, together with (9) and (10), implies that $\left\{\frac{A u\left(t_{1}\right)}{1+t_{1}^{\alpha-1}}: u \in U\right\}$ are equicontinuous on any finite subinterval of $J$.

Now, we are going to prove that for any given $\varepsilon>0$, there exists sufficiently large $\tau>0$, which satisfies

$$
\left\|\frac{A u\left(t_{1}\right)}{1+t_{1}^{\alpha-1}}-\frac{A u\left(t_{2}\right)}{1+t_{2}^{\alpha-1}}\right\| \leq \varepsilon
$$

for all $u \in U$ and $t_{1}, t_{2} \geq \tau$.

Together with (28), we need only to show that for any given $\varepsilon>0$, there exists sufficiently large $\tau>0$ such that

$$
\begin{aligned}
& \| \int_{0}^{t_{1}} \frac{\left(t_{1}-s\right)^{\alpha-1}}{1+t_{1}^{\alpha-1}} f(s, u(s),(T u)(s),(S u)(s)) \mathrm{d} s \\
& \quad-\int_{0}^{t_{2}} \frac{\left(t_{2}-s\right)^{\alpha-1}}{1+t_{2}^{\alpha-1}} f(s, u(s),(T u)(s),(S u)(s)) \mathrm{d} s \|<\varepsilon .
\end{aligned}
$$


It follows from (10) that for any given $\varepsilon>0$, there exists a sufficiently large $L>0$ such that

$$
\int_{L}^{+\infty}\|f(t, u,(T u)(t),(S u)(t))\| \mathrm{d} s<\frac{\varepsilon}{3} \quad \forall u \in U,
$$

and there exists $K>0$ such that

$$
\int_{0}^{+\infty}\|f(t, u,(T u)(t),(S u)(t))\| \mathrm{d} s \leq K \quad \forall u \in U .
$$

On the other hand, let $g(t, s)=\frac{(t-s)^{\alpha-1}}{1+t^{\alpha-1}}, s \in[0, L], t \in[L,+\infty)$, then we have

$$
\lim _{t \rightarrow \infty} \sup _{s \in[0, L]}|g(t, s)-1| \leq \lim _{t \rightarrow \infty} g(t, L)=0 .
$$

Thus, there exists $\tau>0$ such that for $t_{1}, t_{2} \geq \tau$,

$$
\begin{aligned}
& \sup _{s \in[0, L]}\left|g\left(t_{1}, s\right)-g\left(t_{2}, s\right)\right| \\
& \quad \leq \sup _{s \in[0, L]}\left|g\left(t_{1}, s\right)-1\right|+\sup _{s \in[0, L]}\left|g\left(t_{2}, s\right)-1\right| \\
& \quad<\frac{\varepsilon}{3 K} .
\end{aligned}
$$

Therefore, from (29), (30) and (31) we have

$$
\begin{aligned}
& \| \int_{0}^{t_{1}} \frac{\left(t_{1}-s\right)^{\alpha-1}}{1+t_{1}^{\alpha-1}} f(s, u(s),(T u)(s),(S u)(s)) \mathrm{d} s \\
& \quad-\int_{0}^{t_{2}} \frac{\left(t_{2}-s\right)^{\alpha-1}}{1+t_{2}^{\alpha-1}} f(s, u(s),(T u)(s),(S u)(s)) \mathrm{d} s \| \\
& \leq \int_{0}^{L}\left|\frac{\left(t_{1}-s\right)^{\alpha-1}}{1+t_{1}^{\alpha-1}}-\frac{\left(t_{2}-s\right)^{\alpha-1}}{1+t_{2}^{\alpha-1}}\right|\|f(s, u(s),(T u)(s),(S u)(s))\| \mathrm{d} s \\
& \quad+\int_{L}^{t_{1}} \frac{\left(t_{1}-s\right)^{\alpha-1}}{1+t_{1}^{\alpha-1}}\|f(s, u(s),(T u)(s),(S u)(s))\| \mathrm{d} s \\
& \quad+\int_{L}^{t_{2}} \frac{\left(t_{2}-s\right)^{\alpha-1}}{1+t_{2}^{\alpha-1}}\|f(s, u(s),(T u)(s),(S u)(s))\| \mathrm{d} s \\
& \leq \frac{\varepsilon}{3 K} \int_{0}^{L}\|f(s, u(s),(T u)(s),(S u)(s))\| \mathrm{d} s+\frac{\varepsilon}{3}+\frac{\varepsilon}{3}<\varepsilon .
\end{aligned}
$$

Consequently, the proof is complete.

Lemma 2.6 Let assumptions $\left(\mathrm{H}_{1}\right),\left(\mathrm{H}_{2}\right)$ and $\left(\mathrm{H}_{3}\right)$ be satisfied, and let $U$ be a bounded subset of $B C[J, E]$. Then

$$
\alpha_{B}(A U)=\sup _{t \in J} \alpha_{E}\left(\frac{(A u)(t)}{1+t^{\alpha-1}}\right) .
$$

Proof By Lemma 2.2, we know $A U$ is a bounded subset of $B C[J, E]$. Thus,

$$
\varrho=: \sup _{t \in J} \alpha_{E}\left(\frac{(A U)(t)}{1+t^{\alpha-1}}\right)<\infty .
$$


First, we claim that $\alpha_{B}(A U) \leq \varrho$.

In fact, by Lemma 2.5 , we know that for any given $\varepsilon>0$, there exists a $\tau>0$ such that

$$
\left\|\frac{(A u)\left(t_{1}\right)}{1+t_{1}^{\alpha-1}}-\frac{(A u)\left(t_{2}\right)}{1+t_{2}^{\alpha-1}}\right\|<\varepsilon
$$

uniformly with respect to $u \in U$ and $t_{1}, t_{2} \geq \tau$.

Since $\left\{\frac{(A u)(t)}{1+t^{\alpha-1}}: u \in U\right\}$ is equicontinuous on $[0, \tau]$, by Lemma 1.3 , we know

$$
\alpha_{B}\left(\left.A U\right|_{[0, \tau]}\right)=\max _{t \in[0, \tau]} \alpha_{E}\left(\frac{(A u)(t)}{1+t^{\alpha-1}}\right)
$$

where

$$
\left.A U\right|_{[0, \tau]}=\{u(t): t \in[0, \tau], u \in U\},
$$

that is, $\left.A U\right|_{[0, \tau]}$ is the restriction of $A U$ on $[0, \tau]$. Therefore, there exists $U_{1}, U_{2}, \ldots, U_{k} \subset U$ such that

$$
U=\bigcup_{i=1}^{k} U_{i}
$$

satisfying

$$
\left.A U\right|_{[0, \tau]}=\left.\bigcup_{i=1}^{k} A U_{i}\right|_{[0, \tau]}, \quad \operatorname{diam}_{B}\left(A U_{i}\right)<\varrho+\varepsilon, \quad i=1,2,3, \ldots, k
$$

where $\operatorname{diam}_{B}(\cdot)$ denote the diameters of bounded subsets of $B C[J, E]$.

At the same time, for any $A u_{1}, A u_{2} \in A U_{i}$, by (32) and (33), we obtain

$$
\begin{aligned}
\left\|\frac{\left(A u_{1}\right)(t)}{1+t^{\alpha-1}}-\frac{\left(A u_{2}\right)(t)}{1+t^{\alpha-1}}\right\| \leq & \left\|\frac{\left(A u_{1}\right)(t)}{1+t^{\alpha-1}}-\frac{\left(A u_{2}\right)(t)}{1+t^{\alpha-1}}\right\|+\left\|\frac{\left(A u_{1}\right)(t)}{1+t^{\alpha-1}}-\frac{\left(A u_{2}\right)(t)}{1+t^{\alpha-1}}\right\| \\
& +\left\|\frac{\left(A u_{1}\right)(t)}{1+t^{\alpha-1}}-\frac{\left(A u_{2}\right)(t)}{1+t^{\alpha-1}}\right\| \\
\leq & \varepsilon+\varrho+\varepsilon+\varepsilon=\varrho+3 \varepsilon \quad \forall t \in[\tau,+\infty) .
\end{aligned}
$$

It follows from (33) and (34) that

$$
\operatorname{diam}_{B}\left(A U_{i}\right) \leq \varrho+3 \varepsilon, \quad i=1,2,3, \ldots, k .
$$

Then, by using $A U=\bigcup_{i=1}^{k} A U_{i}$, we have

$$
\alpha_{B}(A U) \leq \varrho .
$$

On the other hand, for any given $\varepsilon>0$, there exist $V_{i} \subset U, i=1,2,3, \ldots, l$, such that

$$
A U=\bigcup_{i=1}^{l} A V_{i} \quad \text { and } \quad \operatorname{diam}_{B}\left(A V_{i}\right) \leq \alpha_{B}(A U)+\varepsilon .
$$


Hence, for $\forall t \in J, \forall u_{1}, u_{2} \in U_{i}, i=1,2,3, \ldots, l$, we have

$$
\left\|\frac{\left(A u_{1}\right)(t)}{1+t^{\alpha-1}}-\frac{\left(A u_{2}\right)(t)}{1+t^{\alpha-1}}\right\| \leq\left\|A u_{1}-A u_{2}\right\|_{B} \leq \alpha_{B}(A U)+\varepsilon .
$$

Since $(A U)(t)=\bigcup_{i=1}^{l}\left(A V_{i}\right)(t)$ together with (35), we get

$$
\alpha_{E}\left(\frac{(A u)(t)}{1+t^{\alpha-1}}\right) \leq \alpha_{B}(A U)+\varepsilon
$$

that is,

$$
\sup _{t \in J} \alpha_{E}\left(\frac{(A u)(t)}{1+t^{\alpha-1}}\right) \leq \alpha_{B}(A U)+\varepsilon .
$$

Because $\varepsilon$ is arbitrary, we obtain

$$
\sup _{t \in J} \alpha_{E}\left(\frac{\left(A u_{1}\right)(t)}{1+t^{\alpha-1}}\right) \leq \alpha_{B}(A U)
$$

Consequently, the proof is complete.

\section{Main results}

In this section, we give and prove our main results.

Theorem 3.1 Let $\left(\mathrm{H}_{1}\right)-\left(\mathrm{H}_{6}\right)$ be satisfied. Then BVP (1) has at least two positive solutions $u^{*}, u^{* *} \in B C[J, P]$ such that $u^{*}(t) \gg u_{0}$ for $t \in I$.

Proof By Lemma 2.2 and Lemma 2.4, the operator $A$ defined by (3) is continuous from $B C[J, P]$ into $B C[J, P]$, and by Lemma 2.3 , we need only to show that $A$ has two positive fixed points $u^{*}, u^{* *} \in B C[J, P]$ such that $u^{*}(t) \gg u_{0}$ for $t \in I$.

First, we shall prove $A$ is compact.

Let $U=\left\{u_{n}\right\} \subset B C[J, E]$ be bounded and $\left\|u_{n}\right\| \leq K(n=1,2,3, \ldots)$. From (9), we can choose a sufficiently large $\tau>0$ such that for all $u \in U$

$$
\int_{\tau}^{+\infty}\|f(s, u(s),(T u)(s),(S u)(s))\| \mathrm{d} s<\varepsilon .
$$

It follows from Lemma 2.5 that

$$
\left\{\frac{\left(A u_{n}\right)(t)}{1+t^{\alpha-1}}: n=1,2,3, \ldots\right\}
$$

is equicontinuous on $[0, \tau]$. Thus, by (3), (36) and (37), we have

$$
\begin{aligned}
\alpha_{E}\left(\frac{A U(t)}{1+t^{\alpha-1}}\right) \leq & \frac{1}{\Gamma(\alpha)} \int_{0}^{\tau} \alpha_{E}(f(s, U(s),(T U)(s),(S U)(s))) \mathrm{d} s+2 \varepsilon \\
& +\frac{1}{\Gamma(\alpha)} \sum_{i=1}^{m} \beta_{i} \int_{0}^{\eta_{i}}\left(\eta_{i}-s\right)^{\alpha-1} \alpha_{E}(f(s, U(s),(T U)(s),(S U)(s))) \mathrm{d} s \\
& +\int_{0}^{\tau} \alpha_{E}(f(s, U(s),(T U)(s),(S U)(s))) \mathrm{d} s+2 \lambda \varepsilon,
\end{aligned}
$$


where $\frac{A U(t)}{1+t^{\alpha-1}}=\left\{\frac{A u_{n}(t)}{1+t^{\alpha-1}}: n=1,2,3, \ldots\right\}, U(s)=\left\{u_{n}(s): n=1,2,3, \ldots\right\},(T U)(s)=\left\{\left(T u_{n}\right)(s): n=\right.$ $1,2,3, \ldots\},(S U)(s)=\left\{\left(S u_{n}\right)(s): n=1,2,3, \ldots\right\}$.

Since $U(s),(T U)(s),(S U)(s) \subset P_{r^{*}}$ for $s \in J$, where $r^{*}=\max \left\{r, k^{*} r, h^{*} r\right\}$, we see that, by virtue of assumption $\left(\mathrm{H}_{2}\right)$,

$$
\alpha_{E}(f(s, U(s),(T U)(s),(S U)(s)))=0 \quad \forall t \in J .
$$

It follows from (38) and (39) that

$$
\alpha_{E}\left(\frac{A U(t)}{1+t^{\alpha-1}}\right) \leq 2(1+\lambda) \varepsilon
$$

which implies, by virtue of the arbitrariness of $\varepsilon$, that

$$
\alpha_{E}\left(\frac{A U(t)}{1+t^{\alpha-1}}\right)=0 \quad \forall t \in J .
$$

Using Lemma 2.6, we have

$$
\alpha_{B}(A U)=\sup _{t \in J}\left(\frac{A U(t)}{1+t^{\alpha-1}}\right)=0
$$

Thus, we can conclude that $A U$ is relatively compact in $B C[J, E]$, i.e., $A$ is compact.

As in the proof of Lemma 2.2, (12) holds. Choose

$$
\begin{aligned}
R^{*}> & \left\{2\left\|u_{0}\right\|, 2\left(\frac{1}{\Gamma(\alpha)}+\lambda\right)\left(a^{*}+M b^{*}\right)\right. \\
& \left.+\frac{2 \lambda}{\Gamma(\alpha)} \sum_{i=1}^{m} \beta_{i} \int_{0}^{\eta_{i}}\left(\eta_{i}-s\right)^{\alpha-1}(a(s)+M b(s)) \mathrm{d} s\right\}
\end{aligned}
$$

where $u_{0} \gg \theta$ is given in assumption $\left(\mathrm{H}_{6}\right)$, and let $\Omega_{1}=\left\{u \in B C[J, P]:\|u\|<R^{*}\right\}$. Then $\bar{\Omega}_{1}=\left\{u \in B C[J, P]:\|u\| \leq R^{*}\right\}$ and, by (12) and (40), we have

$$
A\left(\bar{\Omega}_{1}\right) \subset \Omega_{1}
$$

By virtue of $\left(\mathrm{H}_{4}\right)$, there exists an $r_{1}>0$ such that

$$
\begin{aligned}
& \left\|f\left(t,\left(1+t^{\alpha-1}\right) u,\left(1+t^{\alpha-1}\right) v,\left(1+t^{\alpha-1}\right) w\right)\right\| \leq \varepsilon_{2} d(t)(\|u\|+\|v\|+\|w\|) \\
& \forall t \in J, u, v, w \in P,\|u\|+\|v\|+\|w\| \leq r_{1}
\end{aligned}
$$

where

$$
\varepsilon_{2}=\frac{1}{2\left(1+k^{*}+h^{*}\right)}\left[\left(\frac{1}{\Gamma(\alpha)}+\lambda\right) d^{*}+\frac{\lambda}{\Gamma(\alpha)} \sum_{i=1}^{m} \beta_{i} \int_{0}^{\eta_{i}}\left(\eta_{i}-s\right)^{\alpha-1} d(s) \mathrm{d} s\right]^{-1} .
$$

Let

$$
r_{2}=\frac{r_{1}}{1+k^{*}+h^{*}}
$$


Then, for $u \in B C[J, P]$ with $\|u\|_{B} \leq r_{2}$, we have by (42)

$$
\begin{aligned}
& \|f(t, u(t),(T u)(t),(S u)(t))\| \\
& \quad=\left\|f\left(t,\left(1+t^{\alpha-1}\right) \frac{u(t)}{1+t^{\alpha-1}},\left(1+t^{\alpha-1}\right) \frac{(T u)(t)}{1+t^{\alpha-1}},\left(1+t^{\alpha-1}\right) \frac{(S u)(t)}{1+t^{\alpha-1}}\right)\right\| \\
& \quad \leq \varepsilon_{2} d(t)\left(\frac{\|u(t)\|}{1+t^{\alpha-1}}+\frac{\|(T u)(t)\|}{1+t^{\alpha-1}}+\frac{\|(S u)(t)\|}{1+t^{\alpha-1}}\right) \\
& \quad \leq \varepsilon_{2} d(t)\left(1+k^{*}+h^{*}\right)\|u\|_{B} \quad \forall t \in J .
\end{aligned}
$$

It follows from (3), (43) and (44) that

$$
\begin{aligned}
\frac{\|(A u)(t)\|}{1+t^{\alpha-1} \leq} & \frac{1}{\Gamma(\alpha)} \int_{0}^{t}\|f(s, u(s),(T u)(s),(S u)(s))\| \mathrm{d} s \\
& +\frac{\lambda}{\Gamma(\alpha)} \sum_{i=1}^{m} \beta_{i} \int_{0}^{\eta_{i}}\left(\eta_{i}-s\right)^{\alpha-1}\|f(s, u(s),(T u)(s),(S u)(s))\| \mathrm{d} s \\
& +\lambda \int_{0}^{+\infty}\|f(s, u(s),(T u)(s),(S u)(s))\| \mathrm{d} s \\
\leq & \left(\frac{1}{\Gamma(\alpha)}+\lambda\right) \varepsilon_{2} d^{*}\left(1+k^{*}+h^{*}\right)\|u\|_{B} \\
& +\frac{\varepsilon_{2}\left(1+k^{*}+h^{*}\right)}{\Gamma(\alpha)} \sum_{i=1}^{m} \beta_{i} \int_{0}^{\eta_{i}}\left(\eta_{i}-s\right)^{\alpha-1} d(s) \mathrm{d} s \\
= & \frac{1}{2}\|u\|_{B},
\end{aligned}
$$

which implies

$$
\|A u\|_{B} \leq \frac{1}{2}\|u\|_{B}, \quad u \in B C[J, P],\|u\|_{B} \leq r_{2} .
$$

Choose

$$
0<r<\min \left\{\frac{\left\|u_{0}\right\|}{N\left(1+t_{*}^{\alpha-1}\right)}, r_{2}, R\right\} .
$$

Let $\Omega_{2}=\left\{u \in B C[J, P]:\|u\|_{B}<r\right\}$. Then $\bar{\Omega}_{2}=\left\{u \in B C[J, P]:\|u\|_{B} \leq r\right\}$, and we have, by (45) and (46),

$$
A\left(\bar{\Omega}_{2}\right) \subset \Omega_{2}
$$

Let $\Omega_{3}=\left\{u \in B C[J, P]:\|u\|_{B}<R, u(t) \gg u_{0}, t \in I\right\}$, and we are going to show that $\Omega_{3}$ is an open set of $B C[J, P]$. It is clear that we need only to show the following: for any $\bar{u} \in \Omega_{3}$, there exists $\eta>0$ such that $u \in B C[J, P],\|u-\bar{u}\|_{B}<\eta$ implies that $u(t) \gg u_{0}$ for $t \in I$. We have $\bar{u}(t) \gg u_{0}$ for $t \in I$. So, for any $s \in I$, there exists a $\varepsilon=\varepsilon(s)>0$ such that

$$
\bar{u}(s) \geq(1+3 \varepsilon) u_{0} .
$$


Since $u_{0} \gg \theta$ and $\bar{u}(t)$ is continuous on $J$, we can find an open interval $I(s, \delta)=(s-\delta, s+\delta)$ $(\delta>0)$ such that

$$
\varepsilon u_{0}+[\bar{u}(t)-\bar{u}(s)] \geq \theta \quad \forall t \in I(s, \delta),
$$

which implies by virtue of (48) that

$$
\bar{u}(t) \geq(1+2 \varepsilon) u_{0} \quad \forall t \in I(s, \delta) .
$$

Since $I$ is compact, there is a finite collection of such intervals $\left\{I\left(s_{j}, \delta_{j}\right)\right\}(j=1,2, \ldots, k)$ which cover $I$, and

$$
\bar{u}(t) \geq\left(1+2 \varepsilon_{j}\right) u_{0} \quad \forall t \in I\left(s_{j}, \delta_{j}\right)(j=1,2, \ldots, k)
$$

where $\varepsilon_{j}>0(j=1,2, \ldots, k)$. Consequently,

$$
\bar{u}(t) \geq\left(1+2 \varepsilon^{*}\right) u_{0} \quad \forall t \in I
$$

where $\varepsilon^{*}=\min _{1 \leq j \leq k}\left\{\varepsilon_{j}\right\}>0$. Since $u_{0} \gg \theta$, there exists an $\eta=\frac{\left\|u_{0}\right\|}{2 N\left(1+t_{*}^{\alpha}\right)}>0$ such that

$$
\varepsilon^{*} u_{0}+[u(t)-\bar{u}(t)] \geq \theta \quad \forall t \in I,
$$

whenever $u \in B C[J, P]$ satisfying $\|u-\bar{u}\|_{B}<\eta$, which implies by virtue of (49) and (50) that

$$
u(t) \geq\left(1+\varepsilon^{*}\right) u_{0} \gg u_{0}, \quad u \in B C[J, P],\|u-\bar{u}\|_{B}<\eta .
$$

Thus, we have proved that $\Omega_{3}$ is open in $B C[, P]$.

On the other hand, Lemma 2.4 and assumption $\left(\mathrm{H}_{6}\right)$ imply

$$
\begin{aligned}
(A u)(t) & \geq \int_{t_{*}}^{t^{*}} G(t, s) f(s, u(s),(T u)(s),(S u)(s)) \mathrm{d} s \\
& \geq \int_{t_{*}}^{t^{*}} G(t, s) \sigma(s) \mathrm{d} s u_{0} \\
& \geq \int_{t_{*}}^{t^{*}} \gamma(s) \sigma(s) \mathrm{d} s u_{0} \\
& \gg u_{0} \quad \forall t \in I .
\end{aligned}
$$

Hence

$$
A\left(\bar{\Omega}_{3}\right) \subset \Omega_{3} .
$$

Since $\Omega_{1}, \Omega_{2}$ and $\Omega_{3}$ are nonempty bounded convex open subsets of $B C[J, P]$, we see that (41), (47) and (52) imply by virtue of Lemma 1.1 the fixed point indices

$$
i\left(A, \Omega_{i}, B C[J, P]\right)=1 \quad(i=1,2,3) .
$$


On the other hand, for $u \in \Omega_{3}$, we have $u(t) \gg u_{0}$, and so

$$
\|u\|_{B} \geq \frac{\left\|u\left(t_{*}\right)\right\|}{1+t_{*}^{\alpha-1}} \geq \frac{\left\|u_{0}\right\|}{N\left(1+t_{*}^{\alpha-1}\right)} .
$$

Consequently,

$$
\Omega_{2} \subset \Omega_{1} \subset B C[J, P], \quad \Omega_{3} \subset \Omega_{1} \subset B C[J, P], \quad \Omega_{2} \cap \Omega_{3}=\emptyset .
$$

By (53), (54) and the additivity of the fixed point index (Lemma 1.2), we can obtain

$$
\begin{aligned}
& i\left(A, \Omega_{1} /\left(\overline{\Omega_{2} \cup \Omega_{3}}\right), B C[J, P]\right) \\
& \quad=i\left(A, \Omega_{1}, B C[J, P]\right)-i\left(A, \Omega_{2}, B C[J, P]\right)-i\left(A, \Omega_{3}, B C[J, P]\right)=-1 .
\end{aligned}
$$

Finally, (53), (54) and (55) imply that $A$ has two fixed points $u^{*} \in \Omega_{3}$ and $u^{* *} \in$ $\Omega_{1} /\left(\overline{\Omega_{2} \cup \Omega_{3}}\right)$. We have, by $(51), u^{*}(t) \gg u_{0}$ for $t \in I$. The proof is complete.

Remark 3.1 Assumption $\left(\mathrm{H}_{7}\right)$ and the continuity of $f$ imply that $f(t, \theta, \theta, \theta)=\theta$ for $t \in J$. Hence, under the assumptions of the theorem, BVP (1) has the trivial solution $u(t) \equiv \theta$ besides two positive solutions $u^{*}$ and $u^{* *}$.

Theorem 3.2 Let $\left(\mathrm{H}_{1}\right)-\left(\mathrm{H}_{5}\right)$ and $\left(\mathrm{H}_{7}\right)$ be satisfied. Then BVP (1) has at least one positive solution $\tilde{u}(t) \in B C[J, P]$ such that $\tilde{u}(t) \geq u_{0}$ for $t \in I$.

Proof By Lemma 2.2, Lemma 2.4 and the proof of Theorem 3.1, the operator $A$ defined by (3) is completely continuous from $B C[J, P]$ into $B C[J, P]$, and by Lemma 2.3 , we need only to show that $A$ has one positive fixed point $\tilde{u} \in B C[J, P]$ such that $\tilde{u}(t) \gg u_{0}$ for $t \in I$.

As in the proof of Lemma 2.2, (12) holds. Choose $R$ satisfying (40) and let $U=\{u \in$ $\left.B C[J, P]:\|u\| \leq R, u(t) \geq u_{0} \forall t \in I\right\}$, where $u_{0}>\theta$ is given by assumption $\left(\mathrm{H}_{7}\right)$. It is clear that $U$ is a nonempty bounded closed convex subset in $B C[J, P]\left(U \neq \emptyset\right.$ because $\left.2 u_{0} \in U\right)$. Let $u \in U$, by (40), we have $\|A u\| \leq R^{*}$. On the other hand, as in the proof of Theorem 3.1, Lemma 2.4 and assumption $\left(\mathrm{H}_{7}\right)$ imply

$$
\begin{aligned}
(A u)(t) & \geq \int_{t_{*}}^{t^{*}} G(t, s) f(s, u(s),(T u)(s),(S u)(s)) \mathrm{d} s \\
& \geq \int_{t_{*}}^{t^{*}} G(t, s) \sigma(s) \mathrm{d} s u_{0} \\
& \geq \int_{t_{*}}^{t^{*}} \gamma(s) \sigma(s) \mathrm{d} s u_{0} \\
& \geq u_{0} \quad \forall t \in I .
\end{aligned}
$$

Hence, $A u \in W$, and therefore $A U \subset U$. Thus, the Schauder fixed point theorem implies that $A$ has a fixed point $\tilde{u} \in U$, and by (56) $\tilde{u}(t) \geq u_{0}$ for $t \in I$. The proof is complete. 


\section{Conclusion}

In this paper, the issue on the existence of multiple positive solutions of a boundary value problem for $\alpha$-order nonlinear integro-differential equations in a Banach space has been addressed for the first time. Taking advantage of the fixed point index theory of completely continuous operators, the existence conditions for such boundary value problems have been established.

\section{Competing interests}

The authors declare that they have no competing interests.

\section{Authors' contributions}

RL completed the proof and wrote the initial draft. CK provided the problem and gave some suggestions for amendment. $\mathrm{RL}$ then finalized the manuscript. All authors read and approved the final manuscript.

\section{Author details}

${ }^{1}$ College of Information Science and Technology, Donghua University, Shanghai, 201620, China. ${ }^{2}$ College of Fundamental Studies, Shanghai University of Engineering Science, Shanghai, 201620, China. ${ }^{3}$ Department of Applied mathematics, Donghua University, Shanghai, 201620, China.

\section{Acknowledgements}

This work was supported by the Natural Science Foundation of China under grant No. 11271248 and the Science and Technology Research Program of Zhejiang Province under grant No. 2011 C21036.

Received: 25 December 2012 Accepted: 18 March 2013 Published: 8 April 2013

\section{References}

1. Guo, D, Lakshmikantham, V: Nonlinear Problems in Abstract Cones. Academic Press, New York (1988)

2. Guo, D, Lakshmikantham, V, Liu, XZ: Nonlinear Integral Equations in Abstract Spaces. Kluwer Academic, Dordrecht (1996)

3. Arara, A, Benchohra, M, Hamidi, N, Nieto, JJ: Fractional order differential equations on an unbounded domain Nonlinear Anal. 72, 580-586 (2010)

4. Babakhani, A, Gejji, VD: Existence of positive solutions of nonlinear fractional differential equations. J. Math. Anal. Appl. 278, 434-442 (2003)

5. Delbosco, D, Rodino, L: Existence and uniqueness for a nonlinear fractional differential equation. J. Math. Anal. Appl. 204, 609-625 (1996)

6. Diethlm, K, Ford, NJ: Analysis of fractional differential equations. J. Math. Anal. Appl. 265, 229-248 (2002)

7. Sayed, WGE, Sayed, AMAE: On the functional integral equations of mixed type and integro-differential equations of fractional orders. Appl. Math. Comput. 154, 461-467 (2004)

8. Granas, A, Dugundji, J: Fixed Point Theory. Springer, New York (2003)

9. El-Sayed, AMA: On the fractional differential equation. Appl. Math. Comput. 49, 205-213 (1992)

10. Kilbas, AA, Srivastava, HM, Trujillo, JJ: Theory and Applications of Fractional Differential Equations. Elsevier, Amsterdam (2006)

11. Kilbas, AA, Trujillo, JJ: Differential equations of fractional order: methods, results and problems I. Appl. Anal. 78, 153-192 (2001)

12. Kilbas, AA, Trujillo, J: Differential equations of fractional order: methods, results and problems II. Appl. Anal. 81 435-493 (2002)

13. Kosmatov, N: Integral equations and initial value problems for nonlinear differential equations of fractional order. Nonlinear Anal. 70, 2521-2529 (2009)

14. Lakshmikantham, V: Theory of fractional functional differential equations. Nonlinear Anal. 69, 3337-3343 (2008)

15. Lakshmikantham, V, Vatsala, AS: Basic theory of fractional differential equations. Nonlinear Anal. 69, 2677-2682 (2008)

16. Muslim, M, Conca, C, Nandakumaran, AK: Approximate of solutions to fractional integral equation. Comput. Math. Appl. 59, 1236-1244 (2010)

17. Miller, KS, Ross, B: An Introduction to the Fractional Calculus and Fractional Differential Equation. Wiley, New York (1993)

18. Podlubny, I: Fractional Differential Equations: An Introduction to Fractional Derivatives, Fractional Differential Equations, to Methods of Their Solution and Some of Their Applications. Academic Press, San Diego (1999)

19. Stojanović, M: Existence-uniqueness result for a nonlinear $n$-term fractional equation. J. Math. Anal. Appl. 353 244-245 (2009)

20. Samko, SG, Kilbas, AA, Marichev, Ol: Fractional Integrals and Derivatives. Theory and Applications. Gordon \& Breach, Yverdon (1993) 